\title{
The distribution of shower longitudinal profile widths as measured by Telescope Array in stereo mode
}

\author{
Douglas R Bergman $^{* a}$ and Thomas A Stroman ${ }^{a}$ for the Telescope Array \\ Collaboration ${ }^{\dagger}$ \\ ${ }^{a}$ The University of Utah \\ E-mail: bergman@physics.utah.edu \\ E-mail: tastroman@physics.utah.edu
}

\begin{abstract}
Observing UHECR air showers in stereo mode provides a precise measurement of their longitudinal profiles. The Gaisser-Hillas function fits air shower profiles well on average. The range of shower widths can be sensitive to details of average inelasticity and multiplicity in the early part of the shower. Such a measurement can then also be used to constrain the interaction models used in simulating UHECRs. This work can augment the conventional stereo composition measurement. The distribution of the Gaisser-Hillas function FWHM value will be made in bins of energy. These distribution will then be compared to Monte Carlo simulations using the interaction model QGSJetII-03.
\end{abstract}

The 34th International Cosmic Ray Conference,

30 July- 6 August, 2015

The Hague, The Netherlands

\footnotetext{
*Speaker.

${ }^{\dagger}$ Full author list at: http://www.telescopearray.org/images/papers/ICRC2015-authorlist.pdf
} 


\section{Introduction}

The elemental composition of Ultra-High Energy Cosmic Rays (UHECRs) is an important piece of information in understanding the sources of UHECRs. The measurement of the composition is difficult due to the indirect nature of the measurement since experiments at these high energies can only observe the Extensive Air Shower (EAS) produced by UHECR when it encounters Earth's atmosphere.

Traditionally, the depth of shower maximum, $X_{\max }$, has been used as an indicator of the mass of the primary cosmic ray. The use of the correlation between $X_{\max }$ and the mass of the primary particle to determine UHECR composition, however, assumes an idealized shower that grows monotonically due to the cascade process until it reaches shower maximum, and then decreases monotonically due to ionization energy losses and particle absorption. This is not in fact true of all air showers. It is possible that a low-multiplicity first interaction (of the cosmic ray with an air molecule) will result in a significant fraction of the primary's kinetic energy being transferred deeply into the atmosphere. The resulting shower may then appear to have two maxima. While this shower morphology will be rare, the same process should also make some individual showers wider, without actually producing a "double hump". Since the likelihood of widening depends on details of the first interaction, the multiplicity and inelasticity, a measurement of the distribution of shower widths can be used to measure the composition of the primaries, to constrain high-energy interaction models and perhaps even to study the differential cross-section of the first interaction. For UHECRs this provides a means of looking at particle collisions at energies far above what is available in terrestrial accelerators.

The measurement of EAS properties with fluorescence detectors like Telescope Array, is usually done through the use of the Gaisser-Hillas shower function[1],

$$
N(X)=N_{\max }\left(\frac{X-X_{0}}{X_{\max }-X_{0}}\right)^{\frac{X_{\max }-X_{0}}{\Lambda}} e^{\frac{X_{\max }-X}{\Lambda}}
$$

where $N_{\max }$ is the size of the shower at maximum, $X_{\max }$ is the depth (in $\mathrm{g} / \mathrm{cm}^{2}$ ) of shower maximum, $X_{0}$ is an unphysical starting depth for the shower (often negative), and $\Lambda$ controls how fast the shower grows. The function is similar to the Poisson distribution and can be motivated by a simple modification of the Heitler model.

Nowhere in the Gaisser-Hillas function does the width of the shower appear, but it is clear that it must be related to $X_{0}$ and $\Lambda$. We can make this relation concrete, by calculating the full-width half maximum (FWHM) of the function. We first introduce the simplifying notation, $x=\frac{X-X_{0}}{\Lambda}$, $x_{\max }=\frac{X_{\max }-X_{0}}{\Lambda}, y=x / x_{\max }$, and $n=N / N_{\max }$. Using this notation, the Gaisser-Hillas function can be rewritten

$$
n(y)=y^{x_{\max }} e^{x_{\max }-x}
$$

Setting $n=1 / 2$ and taking the natural logarithm of both sides we have

$$
\begin{gathered}
x_{\max } \log y+x_{\max }-x=-\log 2 \\
\log y=(y-1)-\frac{\log 2}{x_{\max }}
\end{gathered}
$$


FWHM, 10 EeV, QGSJetII-03

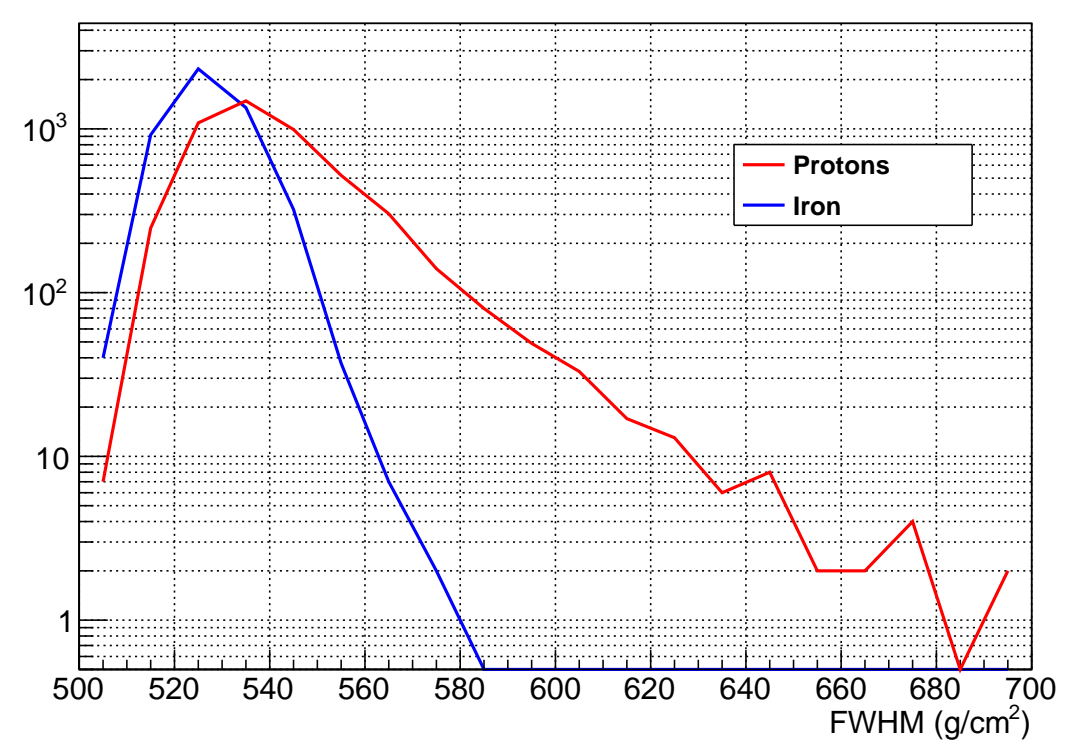

Figure 1: The distribution of widths as seen in showers produced by Corsika using the QGSJetII-03 high energy interaction model for both protons and for iron at $10 \mathrm{EeV}$.

Using the second order expansion of the natural logarithm $\log y \simeq(y-1)-\frac{1}{2}(y-1)^{2}$ we have

$$
(y-1)^{2} \simeq \frac{2 \log 2}{x_{\max }}
$$

Solving for $y$ then (where the Gaisser-Hillas function is half of maximum)

$$
y=1 \pm \sqrt{\frac{\log 4}{x_{\max }}}
$$

The difference between the two solutions is the FWHM: $y_{\mathrm{FWHM}}=2 \sqrt{\log 4 / x_{\max }}$. Plugging back in the usual variables we find

$$
X_{\mathrm{FWHM}}=\sqrt{\Lambda\left(X_{\max }-X_{0}\right) \log 256} \equiv \Sigma_{\mathrm{GH}}
$$

Since the Gaisser-Hillas function has four parameters, we would like to have a fourth parameter mostly uncorrelated to $\Sigma_{\mathrm{GH}}$. We can just take the ratio

$$
R_{\mathrm{GH}} \equiv \frac{\Lambda}{X_{\max }-X_{0}}
$$

Note that $1 / R_{\mathrm{GH}}$ is the exponent in the growth factor of the Gaisser-Hillas function.

An example of the distribution of widths from the QGSJetII-03 model for protons and iron at $10 \mathrm{EeV}$ is shown in Figure 1.

We will use the Gaisser-Hillas function parameterized with these four variables, $N_{\max }, X_{\max }$, $\Sigma_{\mathrm{GH}}$, and $R_{\mathrm{GH}}$, to do the fitting of showers in this analysis. In order to reduce the number of free parameters in our fits, we will be fixing $R_{\mathrm{GH}}$ to the mean value from our Monte Carlo simulations. 


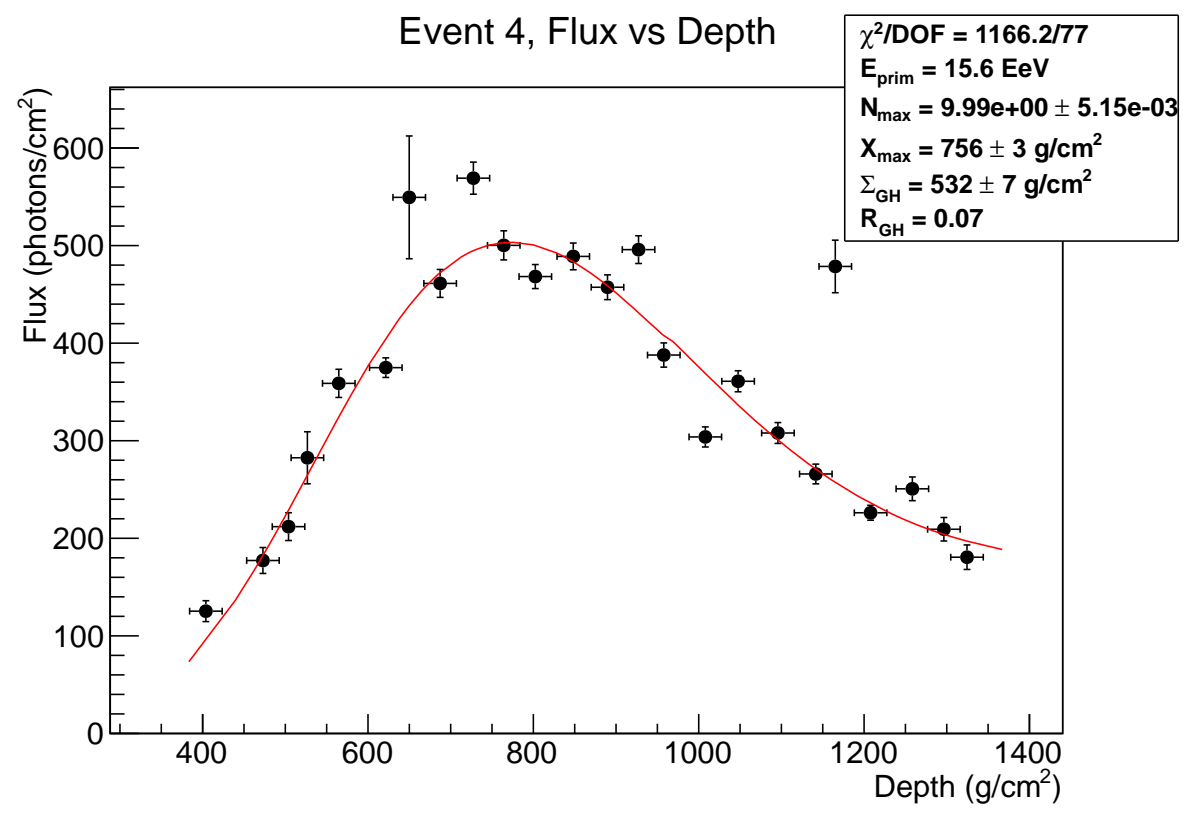

Figure 2: An example shower with profile fit using a variable shower width $R_{\mathrm{GH}}$. While the fit is made to the flux for each tube, the individual tubes have been combined into $40 \mathrm{~g} / \mathrm{cm}^{2}$ bins for plotting purposes.

\section{Data}

The data presented here came from the same data stream and geometry calculation as the TA stereo composition analysis[2]. The determination of shower geometry and location are performed using the same code and identical quality cuts made on these geometrical variables. The filtered data files are in fact identical up to the point of determining the best shower profile. The same weather cut was applied as well. Stereo observations from only two TA sites, Black Rock Mesa and Long Ridge, are used in the width analysis.

The shower profile is reconstructed as in the stereo composition analysis (using an inverse MC approach), except that the Gaisser-Hillas ansatz to be varied is structured to have the parameters $N_{\mathrm{max}}, X_{\mathrm{max}}, \Sigma_{\mathrm{GH}}$, and $R_{\mathrm{GH}} . R_{\mathrm{GH}}$ is held fixed at the value 0.07 (taken from the average of Corsikagenerated showers), while the other three parameters are allowed to vary. An example shower with the fitted width is shown in Figure 2.

In addition to the cuts, applied to stereo $X_{\max }$ analysis, several more cuts are applied to insure a good reconstruction of $\Sigma_{\mathrm{GH}}$ : there must be 40 or more participating PMTs, $100 \mathrm{~g} / \mathrm{cm}^{2}$ or more between the first depth of observation and $X_{\max }$, and the reported uncertainty on the fit value of $\Sigma_{\mathrm{GH}}$ must be less than $20 \mathrm{~g} / \mathrm{cm}^{2}$. Only one site, BRM or LR, was required to pass these cuts. If both sites had a profile measurement of a given shower that passed these cuts, the average value of the fit parameters was used. All profile fits were scanned by eye, and a few $(\sim 5 \%)$ with evidently poor reconstructions (e.g. from clouds) were removed. 
FWHM Distribution, $18.2<\log _{10} \mathrm{E}<18.6$

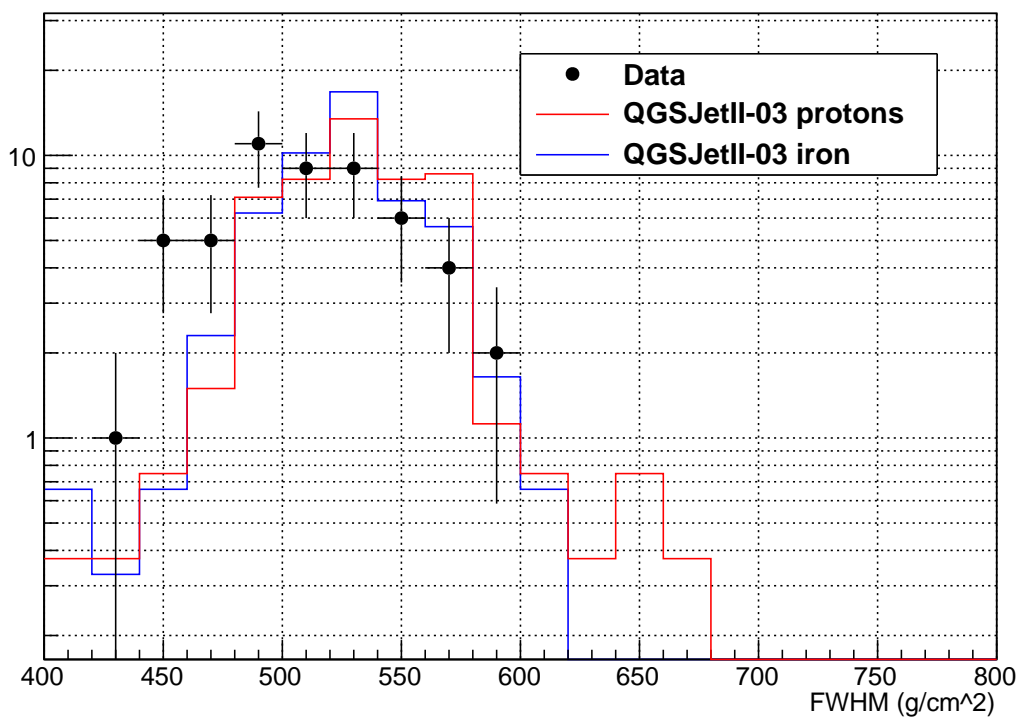

Figure 3: The distribution of $\Sigma_{\mathrm{GH}}$ for $18.2<\log _{10}(E / \mathrm{eV})<18.6$. In addition, normalized distributions of protons (red) and iron (blue) primaries as simulated by QGSJetII-03 are shown.

\section{Simulations}

We simulated a proton and an iron sample using the QGSJetII-03 interaction model. The model was used to make shower libraries of Gaisser-Hillas fit parameters. In simulating the response of the detectors, a set of values was chosen from the library in such a way as to match the HiRes stereo spectrum[3] and isotropic arrival direction. The simulations produce data files which are identical in format to the raw data produced by nature in our detectors. These files are then processed by the same analysis routines as data and subject to the same selection cuts.

\section{Results}

Due to the cuts applied to ensure good reconstruction of $\Sigma_{\mathrm{GH}}$, there is a reduction in the number of events. This allows for only a coarse energy binning. We will show the distribution of $\Sigma_{\mathrm{GH}}$ in three energy bins, for $18.2<\log _{10}(E / \mathrm{eV})<18.6,18.6<\log _{10}(E / \mathrm{eV})<19$, and $19<$ $\log _{10}(E / \mathrm{eV})$. These are shown in Figures 3-5. For comparison we have included the distributions of $\Sigma_{\mathrm{GH}}$ as seen in our MC simulations of proton and iron primaries using the QGSJetII-03 as described above.

\section{Discussion}

Given the cuts required to get a sample of well reconstructed $\Sigma_{\mathrm{GH}}$ values, the TA stereo data set is not big enough to reliably distinguish between different species of UHECR primary. However, it does appear that the QGSJetII-03 model does not do a perfect modeling of the width distribution. In 


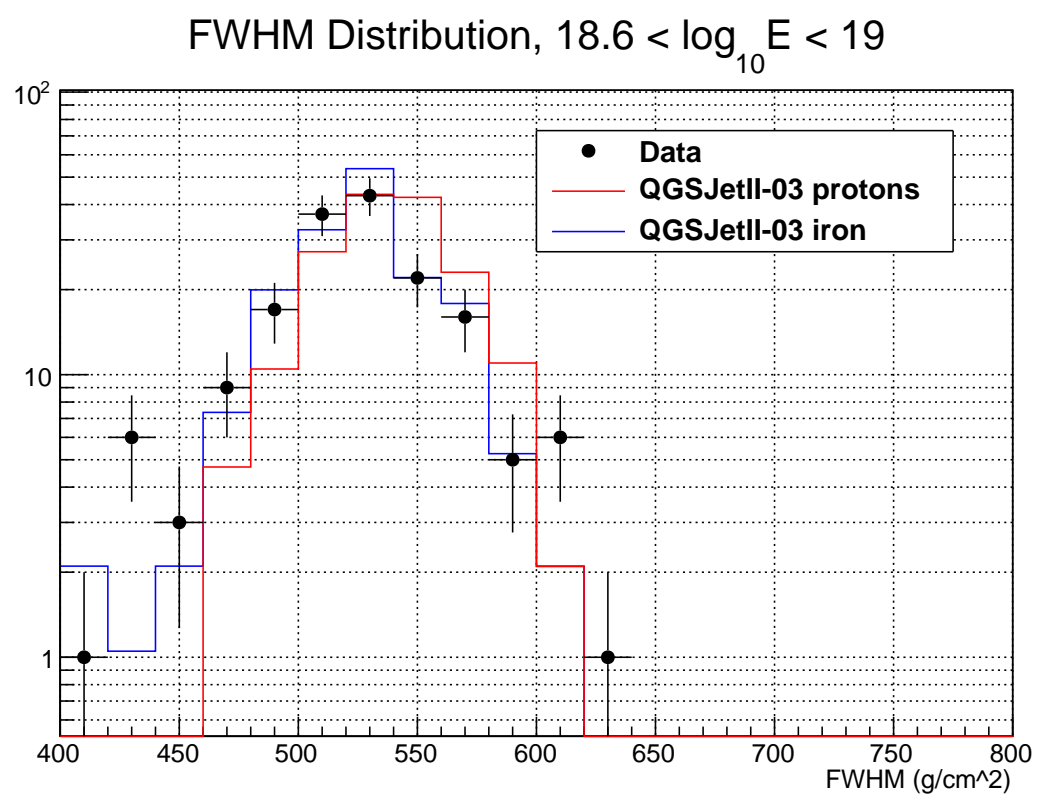

Figure 4: The distribution of $\Sigma_{\mathrm{GH}}$ for $18.6<\log _{10}(E / \mathrm{eV})<19$. In addition, normalized distributions of protons (red) and iron (blue) primaries as simulated by QGSJetII-03 are shown.

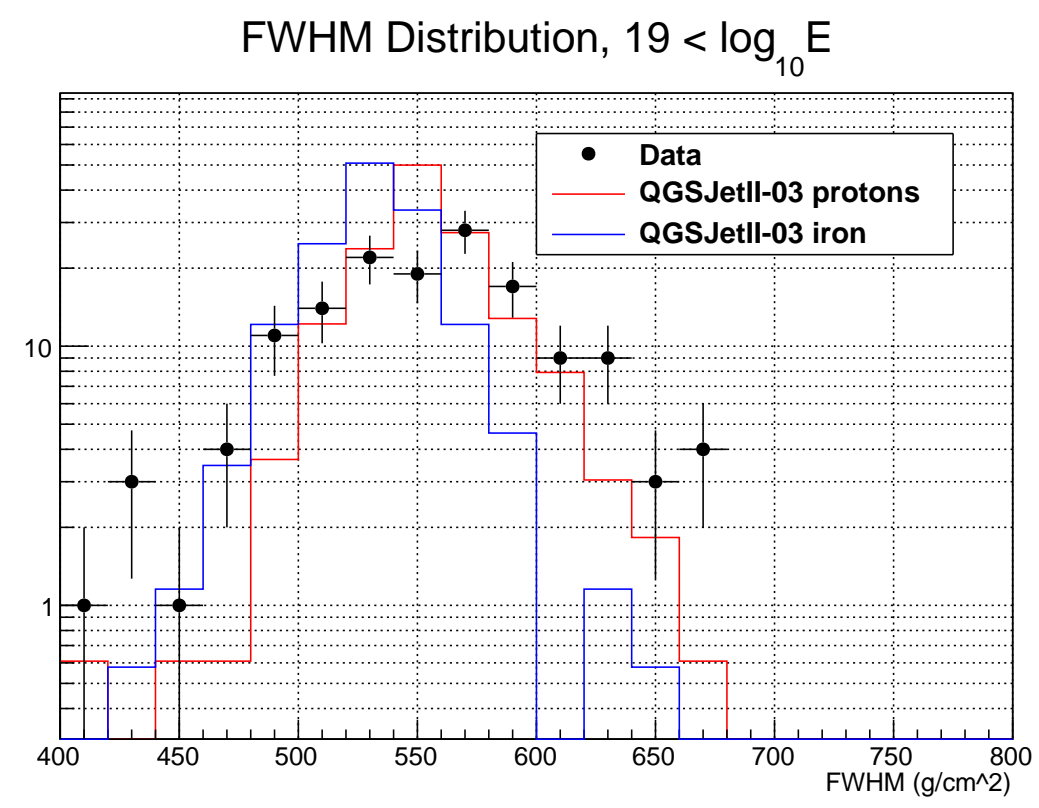

Figure 5: The distribution of $\Sigma_{\mathrm{GH}}$ for $19<\log _{10}(E / \mathrm{eV})$. In addition, normalized distributions of protons (red) and iron (blue) primaries as simulated by QGSJetII-03 are shown. 
the lowest energy bin, shown in Figure 3, the data is lower than both the proton and iron simulations, whereas in the highest energy bin, shown in Figure 5, the data agrees well with the protons and may be a little bit higher. Future work will include more high-energy interaction models to see if this shift is due to interaction model differences or is inherent in the data and analysis we have performed here.

\section{Acknowledgements}

The Telescope Array experiment is supported by the Japan Society for the Promotion of Science through Grants-in-Aid for Scientific Research on Specially Promoted Research (21000002) "Extreme Phenomena in the Universe Explored by Highest Energy Cosmic Rays" and for Scientific Research (19104006), and the Inter-University Research Program of the Institute for Cosmic Ray Research; by the U.S. National Science Foundation awards PHY-0307098, PHY-0601915, PHY-0649681, PHY-0703893, PHY-0758342, PHY-0848320, PHY-1069280, PHY-1069286, PHY1404495 and PHY-1404502; by the National Research Foundation of Korea (2007-0093860, R3210130, 2012R1A1A2008381, 2013004883); by the Russian Academy of Sciences, RFBR grants 11-02-01528a and 13-02-01311a (INR), IISN project No. 4.4502.13, and Belgian Science Policy under IUAP VII/37 (ULB). The foundations of Dr. Ezekiel R. and Edna Wattis Dumke, Willard L. Eccles, and George S. and Dolores Doré Eccles all helped with generous donations. The State of Utah supported the project through its Economic Development Board, and the University of Utah through the Office of the Vice President for Research. The experimental site became available through the cooperation of the Utah School and Institutional Trust Lands Administration (SITLA), U.S. Bureau of Land Management, and the U.S. Air Force. We also wish to thank the people and the officials of Millard County, Utah for their steadfast and warm support. We gratefully acknowledge the contributions from the technical staffs of our home institutions. An allocation of computer time from the Center for High Performance Computing at the University of Utah is gratefully acknowledged.

\section{References}

[1] T.K. Gaisser, A.M. Hillas, Reliability of the method of constant intensity cuts for reconstructing the average development of vertical showers, Proceedings of the 15th ICRC (Plovdiv), 8, (1977) 353.

[2] T.A. Stroman, Y. Tameda, Telescope Array measurement of UHECR composition from stereoscopic fluorescence detection, these proceedings, PoS(ICRC2015)361.

[3] R.U. Abbasi et al., Measurement of the flux of ultra high energy cosmic rays by the stereo technique, Astropart. Phys. 32 (2009) 53. 